\title{
Radial Dupuit interface flow to assess the aquifer storage and recovery potential of saltwater aquifers
}

\author{
Mark Bakker
}

\begin{abstract}
A new accurate numerical solution is presented for aquifer storage and recovery (ASR) systems in coastal aquifers; flow is approximated as radial Dupuit interface flow. The radial velocities of points on the interface are a function of time, the vertical coordinate, and the dimensionless parameter $D$ (the discharge of the well divided by the product of the hydraulic conductivity, the square of the aquifer thickness, and the dimensionless density difference). The recovery efficiency of an ASR system (the ratio of the recovered volume of water divided by the injected volume of water) is determined by $D$ and by the relative lengths of the injection, storage and recovery periods. Graphs are produced for the recovery efficiency as a function of parameter $D$ for ASR operations with and without storage periods and for multiple cycles. The presented solutions and graphs are to be used as screening tools to assess the feasibility of specific injection, storage and recovery scenarios of planned ASR systems in saltwater aquifers without having to run complicated flow and transport models. When the screening tool indicates that recovery efficiencies are acceptable, the consideration of other features such as mixing and chemistry is warranted.
\end{abstract}

Keywords Aquifer storage and recovery . Salt-water/fresh-water relations · Coastal aquifers · Dupuit interface flow

Received: 5 March 2009/Accepted: 20 July 2009

Published online: 11 September 2009

(C) Springer-Verlag 2009

\author{
M. Bakker (®) \\ Water Resources Section, \\ Faculty of Civil Engineering and Geosciences, \\ Delft University of Technology, \\ 2628 CN, Delft, The Netherlands \\ e-mail: mark.bakker@tudelft.nl \\ M. Bakker \\ KWR Watercycle Research Institute, \\ 3433 PE, Nieuwegein, The Netherlands
}

\section{Introduction}

The idea of storing freshwater in a brackish or saltwater aquifer has been around for at least 60 years. Weeks (2002) reports that Cederstrom (1947) was the first within the US Geological Survey (USGS), and possibly the world, to attempt to store freshwater in a brackish aquifer in Virginia. The process of injection, storage, and recovery from an aquifer is nowadays known as "aquifer storage and recovery" under the acronym ASR. This paper considers ASR of freshwater in brackish or saltwater aquifers; for brevity the discussion is about saltwater aquifers even when the salinity is so low that it may qualify for the term brackish.

Recovery of the injected water is never $100 \%$, even in freshwater aquifers. One reason for this is that the injected bubble may drift away from the well due to a regional hydraulic gradient. Another reason is that the quality of part of the injected water may deteriorate as it mixes with the native water (e.g., Lowry and Anderson 2006), or due to chemical reactions between the injected water, native water, and aquifer material; the mobilization of arsenic, for example, has caused significant problems (e.g., Arthur et al. 2002). Other factors that influence the success and sustainability of ASR systems include: the physical properties of the aquifer; the lengths of the injection, storage, and recovery periods; the pumping rates during injection and recovery; and clogging of the well screen.

When freshwater is stored in an aquifer with saltwater, a major complication is caused by the density difference between the injected and native water. The injected freshwater tends to float towards the top of the aquifer as it is lighter than the native saltwater. In an idealized homogeneous freshwater aquifer where mixing is neglected, the interface between the injected and native water remains vertical (left side of Fig. 1). When the native water has a higher density than the injected water, the interface will tilt (right side of Fig. 1). During recovery, the toe of the interface will reach the well screen before all freshwater is recovered from the aquifer. Once the toe of the interface reaches the well screen, the pump is turned off to avoid pumping both fresh and saltwater; the freshwater remaining in the aquifer is considered unrecoverable. This may result in a significant reduction of the recovery efficiency, i.e., the fraction of the volume of injected freshwater that is recovered during a cycle. During long periods of storage (and while still neglecting mixing) the injected freshwater may move 


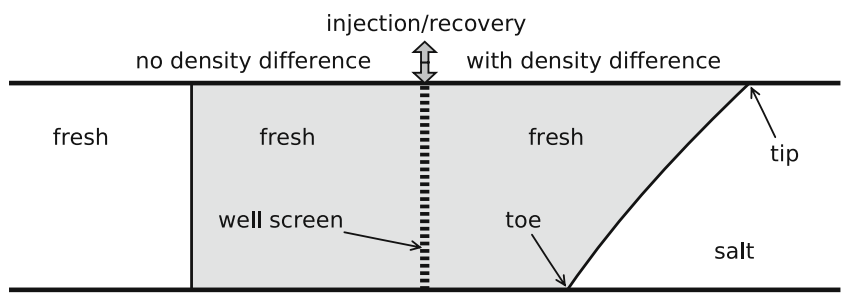

Fig. 1 Vertical cross-section through an ASR well. Without density differences, the interface between the injected and native water remains vertical (left). When the density of the injected water is smaller than the native water, the interface will tilt (right). Dashed line depicts the well screen

entirely to the top of the aquifer where it floats as a pancake on top of the native saltwater, reducing the recovery efficiency to zero.

The body of literature on variable density flow and interface flow is large, and is not reviewed here. Extensive overviews are given in, e.g., Bear et al. (1999) and Cheng and Ouazar (2004), while recent advances are reported in the SWIM proceedings (SWIM 2009). A review of density effects in ASR systems is given in Ward et al. (2007). A description of practical applications was given by, e.g., Reese (2002), who presents an inventory of ASR systems in southern Florida. Ward et al. (2008) performed a numerical study of ASR in a layered aquifer system; one of their simulations is used for a comparison later in this paper.

The objective of this paper is to present screening tools to assess the feasibility of injection, storage and recovery scenarios for ASR systems in saltwater aquifers, without having to use a complicated flow and transport model. The approach considers radial Dupuit interface flow with density effects. When the screening tools indicate that the envisioned scenarios are feasible, it is worthwhile to consider other important factors, including mixing and chemistry.

\section{Dupuit interface flow}

Consider radial interface flow to or from a well in a homogeneous, horizontal, confined aquifer. The interface separates freshwater with density $\rho_{\mathrm{f}}$ from saltwater with density $\rho_{\mathrm{s}}$. A cylindrical coordinate system is adopted with horizontal radial coordinate $r$ and vertical coordinate $z$; the origin is at the bottom of the aquifer at the center of the well. The elevation of the interface as a function of $r$ and time $t$ is written as $y(r, t)$ (Fig. 2). The radial component of the specific discharge vector $q_{r}$ in both the freshwater and saltwater may be written in terms of the equivalent freshwater head $\phi$ (e.g., Bakker 1998; Post et al. 2007)

$q_{r}=-k \frac{\partial \phi}{\partial r}$

where $k$ is the horizontal hydraulic conductivity of freshwater and viscosity differences are neglected. Viscosity differences only become important when the saltwater is much saltier than seawater and may be considered a brine. Aquifers with brine are poor candidates for ASR systems because of the high density contrast, and are not considered here.

The Dupuit approximation is adopted: the resistance to flow in the vertical direction is neglected. The Dupuit approximation gives accurate results for many cases of interface flow (e.g., Chan Hong et al. 1989; Bakker et al. 2004). Under the Dupuit approximation, the head in the aquifer is approximated as hydrostatic, so that the equivalent freshwater head $\phi$ may be expressed in terms of the head $h$ in the freshwater as (recall that $y(r, t)$ is the elevation of the interface)

$\phi=h \quad z \geq y$

$\phi=h+v(y-z) \quad z \leq y$

where $v$ is the dimensionless density difference

$v=\left(\rho_{\mathrm{s}}-\rho_{\mathrm{f}}\right) / \rho_{\mathrm{f}}$

Substitution of Eq. (2) for $\phi$ in Eq. (1) gives

$q_{r}=-k \frac{\partial h}{\partial r} \quad z \geq y$

$q_{r}=-k \frac{\partial h}{\partial r}-k v \frac{\partial y}{\partial r} \quad z \leq y$

Comprehensive radial flow in the aquifer, $Q_{r}$, is obtained through integration of $q_{r}$ over the aquifer thickness, which gives

$Q_{r}=-k H \frac{\partial h}{\partial r}-k v y \frac{\partial y}{\partial r}$

where $H$ is the aquifer thickness. This is the radial flow equivalent of the equations given in Bakker (1998).
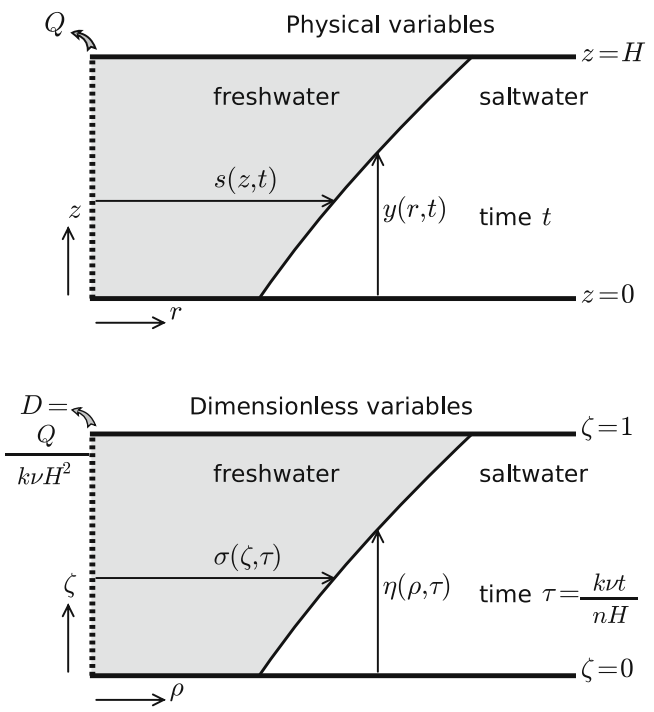

Fig. 2 Radial interface flow problem definition in physical variables and dimensionless variables 


\section{Vertical velocity of the interface}

Consider radial flow caused by a fully penetrating well in a horizontal confined aquifer. The head and flow in the aquifer react quickly to changes in the discharge of the well, as the elastic storage coefficient of confined aquifers is generally small. The interface reacts much slower, so that it is reasonable to neglect elastic storage: the head and flow in the aquifer are approximated to react instantaneously to changes in the discharge of the well or changes in the position of the interface (reasonableness of this approximation is demonstrated in the Example section). The mass balance is replaced by a volume balance (the Boussinesq-Oberbeck approximation, e.g., Holzbecher 1998, p. 32), which is accurate when the differences in salinity are small, as is the case for freshwater and seawater. Hence, at any point in time, the comprehensive radial flow may be written as the flow caused by the well

$Q_{r}(r, t)=-\frac{Q(t)}{2 \pi} \frac{1}{r}$

where $Q(t)$ is the discharge of the well as a function of time (positive for extraction).

Equations (5), (7), and (8) may be combined to eliminate the head-derivative and $Q_{\mathrm{r}}$ from the equations, which gives for $q_{r}$ in the freshwater zone

$q_{r}=-\frac{Q}{2 \pi H} \frac{1}{r}+\frac{k v}{H} y \frac{\partial y}{\partial r} \quad z \geq y$

A similar equation may be obtained for flow in the saltwater, but that is of no further use here.

The vertical component of flow may be obtained through application of continuity of flow (e.g., Strack 1995; Bakker 1998) in cylindrical coordinates

$\frac{1}{r} \frac{\partial\left(r q_{r}\right)}{\partial r}+\frac{\partial q_{z}}{\partial z}=0$

which gives the following integral for the vertical component of flow in the freshwater zone

$q_{z}(r, z)=-\int_{H}^{z} \frac{1}{r} \frac{\partial\left(r q_{r}\right)}{\partial r} d z \quad y \leq z \leq H$

Note that at the top of the aquifer $(z=H)$, the vertical component of flow is set to zero. Substitution of Eq. (9) for $q_{r}$ and working out the integral gives

$q_{z}=-\frac{k v(y-z)}{H}\left[\frac{y}{r} \frac{\partial y}{\partial r}+\left(\frac{\partial y}{\partial r}\right)^{2}+y \frac{\partial^{2} y}{\partial r^{2}}\right] \quad y \leq z \leq H$
An expression for the vertical velocity of the interface may be written as (e.g., Bakker 1998)

$\frac{\partial y}{\partial t}=-v_{r}^{+} \frac{\partial \mathrm{y}}{\partial r}+v_{z}^{+}$

where $v_{r}^{+}$and $v_{z}^{+}$are the radial and vertical components of the freshwater velocity at the interface, obtained from Eqs. (9) and (12) through substitution of $z=H$ and division by the porosity $n$. Combination of Eqs. (9), (12), and (13) gives

$$
\begin{aligned}
\frac{\partial y}{\partial t}= & \frac{Q}{2 \pi n H} \frac{1}{r} \frac{\partial y}{\partial r}-\frac{k v}{n H} \times \\
& {\left[\frac{(y-H) y}{r} \frac{\partial y}{\partial r}+(2 y-H)\left(\frac{\partial y}{\partial r}\right)^{2}+(y-H) y \frac{\partial^{2} y}{\partial r^{2}}\right] }
\end{aligned}
$$

Four dimensionless variables are introduced: the dimensionless radial coordinate $\rho$, the dimensionless vertical coordinate $\zeta$, dimesionless time $\tau$, and the dimensionless vertical position of the interface $\eta$ (see Fig. 2)

$\rho=\frac{r}{H} \quad \zeta=\frac{z}{H} \quad \tau=\frac{k v t}{n H} \quad \eta=\frac{y}{H}$

The dimensionless parameter $D$ is defined as

$D=\frac{Q}{k v H^{2}}$

Parameter $D$, which governs the flow in the ASR system, is the ratio of the discharge of the well to the aquifer properties and the dimensionless density difference. Nordbotten and Celia (2006) used a similar dimensionless grouping in their approximate similarity solution for the injection of carbon dioxide in deep saline aquifers. Ward et al. (2007) defined a dimensionless parameter which they termed the mixed convection ratio, similar to Eq. (16) but $H^{2}$ was replaced by $H R_{\mathrm{b}}$ where $R_{\mathrm{b}}$ is the radius of the freshwater bubble in the absence of density differences. The mixed convection ratio was used to characterize systems, but was not the result of a mathematical derivation. Here, it is shown that the recovery efficiency of the ASR system is determined by the value of $D$.

Writing the vertical velocity of the interface Eq. (14) in dimensionless form gives

$$
\begin{aligned}
\frac{\partial \eta}{\partial \tau}= & \left(\frac{D}{2 \pi}-\eta(\eta-1)\right) \frac{1}{\rho} \frac{\partial \eta}{\partial \rho}-(2 \eta-1)\left(\frac{\partial n}{\partial \rho}\right)^{2} \\
& -\eta(\eta-1) \frac{\partial^{2} \eta}{\partial \rho^{2}}
\end{aligned}
$$

Note that the dimensionless vertical velocity of the interface, in terms of the dimensionless radial distance $\rho$ 
and dimensionless time $\tau$, is a function of only one dimensionless parameter $D$ Eq. (16).

The equation for the vertical velocity of the interface may be further simplified by introducing the similarity variable $u$ defined as

$u=\rho^{2} / \tau$

Writing Eq. (17) in terms of the similarity variable $u$ gives

$$
\begin{aligned}
\frac{d \eta}{d u} & +\left(\frac{D}{\pi}-4 \eta(\eta-1)\right) \frac{1}{u} \frac{d \eta}{d u}-4(2 \eta-1)\left(\frac{d \eta}{d u}\right)^{2} \\
& -4 \eta(\eta-1) \frac{d^{2} \eta}{d u^{2}}=0
\end{aligned}
$$

Equation (19) is an ordinary, non-linear differential equation for $\eta(u)$, the vertical position of the interface as a function of $\rho^{2} / \tau$. This means that solutions for the same dimensionless group $D$, and starting from the same initial position, always result in the same curve when $\eta$ is plotted against $u$. This is examined in more detail later.

\section{Radial velocity of the interface}

The movement of the interface may be simulated through numerical integration of Eq. (17), but a much easier solution is obtained when the dependent variable is the radial position of the interface $s$ rather than the vertical position $y$ (Fig. 2). An equation for the radial velocity of the interface is derived here; numerical integration of either the vertical or radial velocity is discussed in the next section. In dimensionless form, the dimensionless radial position of the interface $\sigma$ is a function of the dimensionless vertical coordinate $\zeta$ and dimensionless time. The equation for the vertical velocity of the interface Eq. (17) may be converted into an equation for the radial velocity of the interface using the following identities (a derivation of these relationships is given at the end of the paper (see Appendix))

$$
\begin{aligned}
& \frac{\partial \eta}{\partial \tau}=-\frac{\partial \sigma}{\partial \tau} \frac{\partial \eta}{\partial \rho} \\
& \frac{\partial \eta}{\partial \rho}=\left(\frac{\partial \sigma}{\partial \zeta}\right)^{-1} \\
& \frac{\partial^{2} \eta}{\partial \rho^{2}}=-\left(\frac{\partial \sigma}{\partial \zeta}\right)^{-3} \frac{\partial^{2} \sigma}{\partial \zeta^{2}}
\end{aligned}
$$

Substitution of these identities in Eq. (17) gives the following equation for the radial velocity

$$
\begin{aligned}
\frac{\partial \sigma}{\partial \tau}= & {\left[\frac{-D}{2 \pi}+\zeta(\zeta-1)\right] \frac{1}{\sigma}+(2 \zeta-1)\left(\frac{\partial \sigma}{\partial \zeta}\right)^{-1} } \\
& -\zeta(\zeta-1)\left(\frac{\partial \sigma}{\partial \zeta}\right)^{-2} \frac{\partial^{2} \sigma}{\partial \zeta^{2}}
\end{aligned}
$$

The radial velocity of the tip $(\zeta=1)$ and toe $(\zeta=0)$ of the interface may be computed explicitly from Eq. (21) as

$$
v_{\text {tip }}=\frac{-D}{2 \pi} \frac{1}{\sigma}+\left(\frac{\partial \sigma}{\partial \zeta}\right)^{-1} v_{\text {toe }}=\frac{-D}{2 \pi} \frac{1}{\sigma}-\left(\frac{\partial \sigma}{\partial \zeta}\right)^{-1}
$$

The velocities of the tip and toe reveal some of the behavior of the interface during injection and recovery. First, recall that the slope of the interface is always positive and that $D$ is negative for injection. The radial position of the tip of the interface is always larger than the radial position of the toe of the interface, and thus the magnitude of the first term on the right-hand side of $v_{\text {tip }}$ is always smaller than the equivalent term of $v_{\text {toe }}$. Hence, during injection, the tilting of the interface due to density differences is mitigated by the injection of water. The reverse happens during recovery, when the tilting of the interface is enhanced because both terms on the right-hand side of $v_{\text {toe }}$ are negative and cause a velocity from the toe toward the well screen.

\section{Numerical simulation of the movement of the interface}

For a numerical simulation, the interface is discretized into a number of points. The movement of the interface may be simulated through numerical integration of either the vertical velocity Eq. (17) or the radial velocity Eq. (21). When integrating the vertical velocity, the boundaries of the model domain (the tip and toe of the interface) are moving radially, while all other points of the interface move in both the radial and vertical directions. Solutions may be obtained by using a moving grid, or with a fixed grid in combination with an algorithm that tracks the movement of the tip and toe of the interface (e.g., Bakker 2003). The main advantage of integrating the radial velocity is that the boundaries of the domain are fixed to the top and bottom of the aquifer, so that a fixed grid may be used without any additional tracking algorithms; this latter approach is used here.

The radial velocity of the interface Eq. (21) is written as

$$
\frac{\partial \sigma}{\partial \tau}=A(\zeta, \sigma, D)+B(\zeta, \sigma) \frac{\partial^{2} \sigma}{\partial \zeta^{2}}
$$

where

$$
\begin{aligned}
& A=\left[\frac{-D}{2 \pi}+\zeta(\zeta-1)\right] \frac{1}{\sigma}+(2 \zeta-1)\left(\frac{\partial \sigma}{\partial \zeta}\right)^{-1} \\
& B=\zeta(\zeta-1)\left(\frac{\partial \sigma}{\partial \zeta}\right)^{-2}
\end{aligned}
$$


Equation (23) is the one-dimensional diffusion equation, where $B$ is the pseudo-diffusion coefficient and $A$ is a pseudo-source term. The interface movement is computed using an implicit finite difference scheme in combination with a predictor-corrector procedure to estimate accurate values of $A$ and $B$ during a time step; an explicit scheme cannot be used as it produces unstable numerical results. A fixed vertical grid is used with $N$ evenly spaced points. At time $\tau, A$ and $B$ are evaluated using a central difference scheme for the derivatives. The radial position of the interface at time $\tau+\Delta \tau$ is estimated by solving Eq. (23) using a fully implicit, central in space finite difference scheme, where $A$ and $B$ are treated as constants. This first estimate of the location of the interface at time $\tau+\Delta \tau$ is used as the predictor step. The parameters $A$ and $B$ are evaluated at the predictor step. Corrected values for $A$ and $B$ during the time from $\tau$ to $\tau+\Delta \tau$ are computed as the average of the values at $\tau$ and $\tau+\Delta \tau$. Using the corrected values of $A$ and $B$, the new radial position of the interface at $\tau+\Delta \tau$ is computed with the same finite difference scheme.

The described predictor-corrector approach results in a stable solution; the accuracy of the solution is determined by the vertical discretization and the time step. The time step is chosen as follows. The radial velocities of all points are estimated with Eq. (23) using standard central difference schemes for the spatial derivatives. Note that the variable $B$ equals zero at the top $(\zeta=1)$ and bottom $(\zeta=0)$ of the aquifer, so the second derivative doesn't have to be computed for the first and last points. Using this estimate of the radial velocity, the time step is chosen such that the radial distance between any two adjacent points does not increase more than $\alpha$ percent. The accuracy of the volume balance is a function of the time step, and thus of the factor $\alpha$. Accurate volume balances (an error of less than $0.1 \%$ for an entire simulation) were obtained with $\alpha=5 \%$ while discretizing the interface in 100 points. Smaller values of $\alpha$ gave errors in the volume balance of $0.01 \%$ and less.

The dimensionless volume $V$ of the freshwater in the aquifer is obtained through integration. The radial position of the interface between grid points $i$ and $i+1$ is written as a linear function

$\sigma=\sigma_{i}+\frac{\Delta \sigma_{i}}{\Delta \zeta} \zeta$

where $\Delta \sigma_{i}=\sigma_{i+1}-\sigma_{i}$ and $\Delta \zeta$ is the vertical grid spacing. The volume $V_{i}$ of freshwater between $\zeta_{i}$ and $\zeta_{i+1}$ is

$V_{i}=\pi \int_{0}^{\Delta \zeta}\left(\sigma_{i}+\frac{\Delta \sigma_{i}}{\Delta \zeta} \zeta\right)^{2} \delta \zeta$

Carrying out the integration and summing the volumes of all the sections gives for the total volume

$$
V=\pi \Delta \zeta \sum_{\mathrm{i}=1}^{N-1}\left(\sigma_{i}^{2}+\sigma_{i} \Delta \sigma_{i}+\left(\Delta \sigma_{i}\right)^{2} / 3\right)
$$

The recovery efficiency is commonly defined as

$\mu_{\mathrm{eff}}=\frac{V_{\text {rec }}}{V_{\mathrm{inj}}}$

where $V_{\text {inj }}$ is the injected volume of water during a cycle and $V_{\text {rec }}$ is the recovered volume during a cycle.

\section{Example}

As a first example, consider a 20 meter thick aquifer with a hydraulic conductivity of $10 \mathrm{~m} / \mathrm{d}$ and a porosity of 0.3 . The density of the native water in the aquifer is $1,025 \mathrm{~kg} /$ $\mathrm{m}^{3}$. Freshwater is injected in the aquifer at a rate of $2,000 \mathrm{~m}^{3} /$ day for 15 days. The dimensionless parameter is $D=20$, and the dimensionless injection time is $\tau_{\mathrm{inj}}=0.625$. The radius of the well is $0.2 \mathrm{~m}$. The initial interface is linear and extends from the radius of the well at the bottom of the aquifer to twice the radius of the well at the top of the aquifer; the initial shape of the interface cannot be vertical as this causes infinite velocities at the tip and toe (Verruijt 1980). After the injection period, water is recovered at the same rate until the toe of the interface reaches the well screen (there is no storage phase in this example). The position of the interface at the end of the first cycle of injection (solid line) and at the end of the first cycle of recovery (dashed line) is shown in Fig. 3; the recovery efficiency of the first cycle is $43 \%$.

Since the same rates are used during injection and recovery, the recovery time only lasts $43 \%$ of 15 days. During the remainder of the 15 days of the recovery phase, the pumping rate is set to zero and the remaining freshwater floats slowly upward at the well and sideways at the tips. For the current example, the movement of the interface is small during this idle period. In fact, the dashed lines in Fig. 3 represent the interface at the end of this idle period; the small amount that the interface moved upward at the well is not noticeable in the figures. It is emphasized that it is not necessary to have an idle period, but it is common in practice. An idle period is often needed to wait for the availability of freshwater to inject. The described process is repeated ten times. The recovery efficiency increases to $70 \%$ for cycle 5 and $75 \%$ for cycle 10 (see Fig. 3).

The recovery efficiency will decrease when the injection period is followed by a period of storage. As an example, the injection period of 15 days is followed by a storage period of 15 days. During this period, the well is not pumped, and the interface tilts due to density differences. The results are shown in Fig. 4, where the lines with short dashes are the positions of the interface at the ends of the storage periods. The tilting is more pronounced when the interface is steeper, and thus the storage phase has a larger effect on earlier cycles than on later cycles. When compared to the case without storage, the recovery efficiency has decreased from 43 to only $23 \%$ for the first cycle, from 70 to $57 \%$ for the fifth cycle, and from 75 to $65 \%$ for the tenth cycle. 

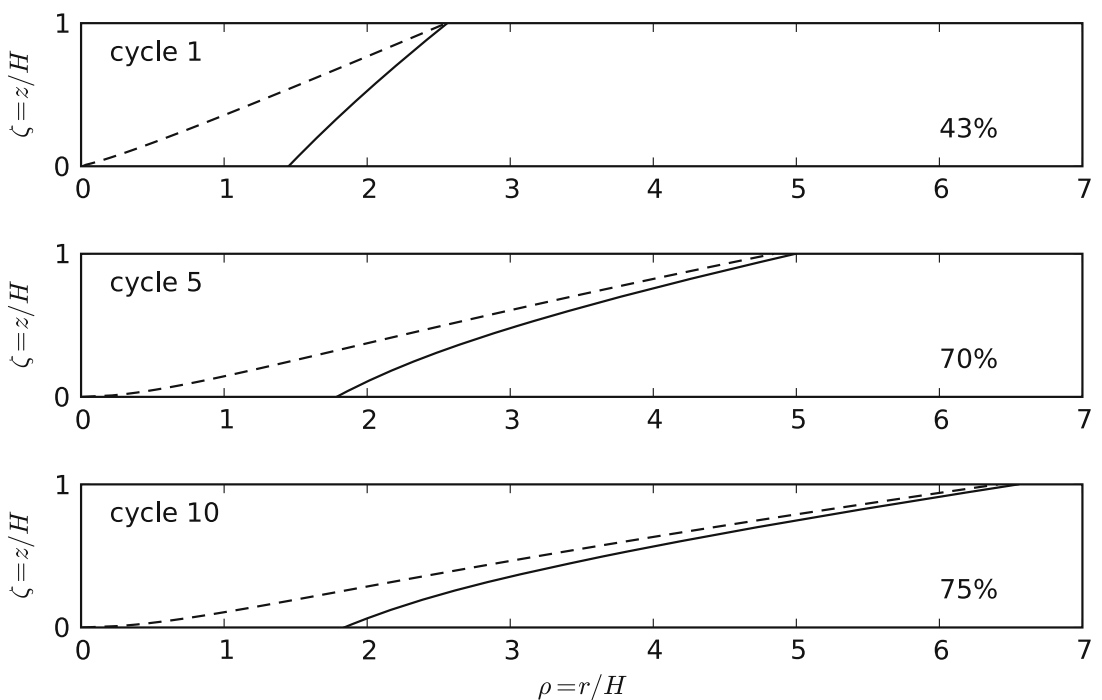

Fig. 3 Position of the interface at end of injection phase (solid lines) and at end of recovery phase (dashed lines) for $D=20$ for cycles 1,5 , and 10 and no storage phase

Elastic storage was neglected in the derivation of the velocity of the interface. The reasonableness of this approximation may be evaluated by computing the time it takes for the flow of a well to approach steady state. Comprehensive steady radial flow to a well in a homogeneous aquifer is obtained from Eq. (8) when the discharge $Q$ is constant. Comprehensive transient radial flow to a well in a homogeneous aquifer is obtained from the Theis solution as (e.g., Strack 1989, section 16).

$Q_{r}=-\frac{Q}{2 \pi} \frac{\exp \left[-S_{\mathrm{s}} r^{2} / 4 k t\right]}{r}$

where $S_{\mathrm{s}}$ is the specific storage of the aquifer, the well starts at $t=0$, and there is no flow initially. Transient flow reaches $95 \%$ of the steady value when the exponential function equals 0.95 and thus

$t=-\frac{S_{\mathrm{s}} r^{2}}{4 k \ln (0.95)}$

For the values used in this example, and with $S_{\mathrm{S}}=$ $0.0001 \mathrm{~m}^{-1}, 95 \%$ of the steady value is reached after 0.02 days at a distance of one aquifer thickness from the well. At a distance of 10 times the aquifer thickness, it takes 2 days to reach $95 \%$ of the steady value. Hence, the approximation seems reasonable, but may cause small inaccuracies farther away from the well during later cycles.

\section{Recovery efficiency as function of parameter D}

It is important to note that the recovery efficiency is not a function of the length of the injection period. When the computations of Fig. 3 are repeated with an injection period of 150 days instead of 15 days, the recovery efficiency of the first cycle is still $43 \%$ and the recovery efficiency of the tenth cycle is still $75 \%$. This conclusion is based on the fact that both the vertical velocity Eq. (17) of the interface and the radial velocity Eq. (21) are a function only of the dimensionless parameter $D$ Eq. (16). This is confirmed by the numerical calculations.

The position of the interface after 15 days of injection is, of course, different from the position of the interface after 150 days of injection. It was shown that the vertical velocity is a function of the similarity variable $u$ Eq. (19). This means that when the elevation of the interface is plotted against $r^{2} / t$, the interface elevation at the end of an injection of 15 days is identical to the interface elevation at the end of an injection of 150 days, provided that the same value of $D$ is used. This holds for the position during injection, storage, and recovery, and for every consecutive cycle. Hence, it is possible to compute a curve that represents the recovery efficiency as a function of the dimensionless parameter $D$, independent of the actual period of injection, but only dependent on the relative lengths of the injection, storage, and recovery periods. The physical parameters of the problem were varied over a wide range of reasonable values (Table 1); variation of the porosity is listed but has no effect on the recovery efficiency. Combination of the appropriate minimum and maximum values of these parameters showed that all possible combinations are covered when $D$ is varied from 0.05 to 40,000 . Values of $D$ below 2.5 gave a recovery efficiency below $5 \%$ on the first cycle while values of $D$ above 1,000 gave a recovery efficiency of more than $90 \%$. Between these values, the recovery efficiency for the first cycle is plotted as a function of $D$ with the solid line in Fig. 5. The dashed line represents the recovery efficiency for cycle 5 , and the line with short dashes the recovery efficiency for cycle 10.

Curves for cases that include a period of storage can also be computed, since the recovery efficiency is a function only of the parameter $D$. Curves were computed for the case that the injection period is followed by a 

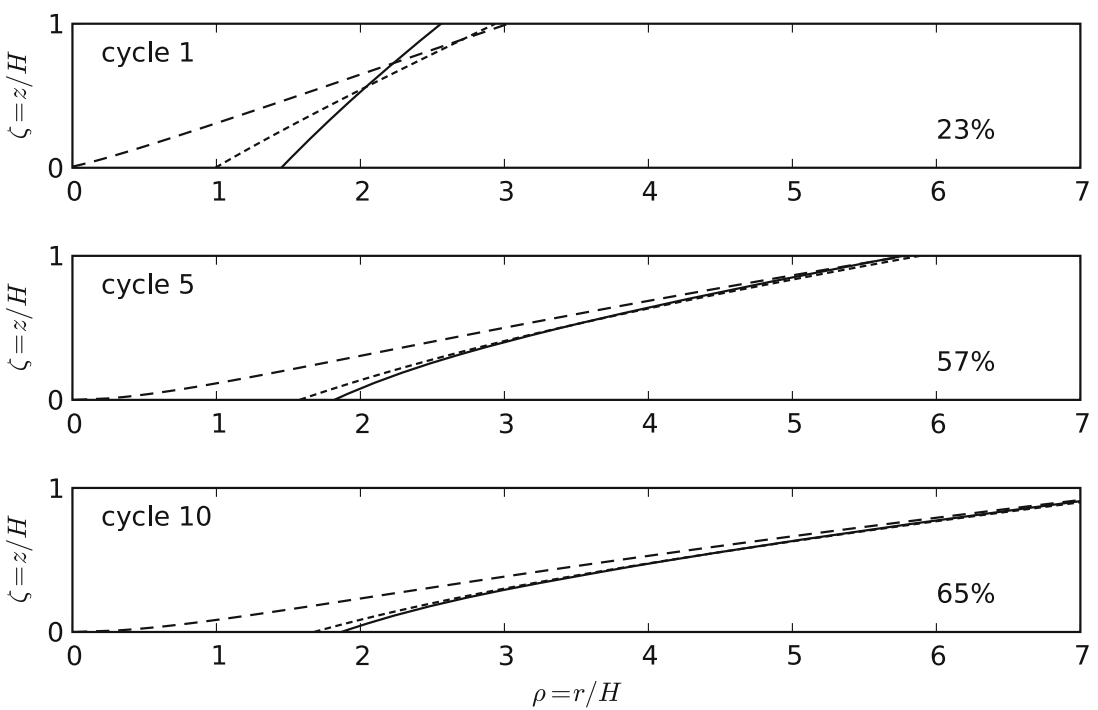

Fig. 4 Position of the interface at end of injection phase (solid lines), at end of storage phase (short dashes) and at end of recovery phase (long dashes) for $D=20$ for cycles 1,5 , and 10; the storage phase is equal in length to the injection phase

storage period of the same length, and for a storage period that is twice the length of the injection phase. The results for the first and fifth cycle are shown in Fig. 6.

\section{A comparison to a transport solution}

The presented solution is compared to one of the solutions presented in Ward et al. (2008), abbreviated to WEA in this section, who performed a numerical study to investigate the effect of a layered aquifer on an ASR system. They did not perform an interface study, but solved the coupled flow and transport equations using the finite element method for radially symmetric cases. They stopped recovery when the salt concentration at the bottom of the well had reached a threshold value. The recovery efficiencies computed with the approach presented in this paper are compared to the values obtained by WEA, as reported in their Fig. 9 for the homogeneous case. This example concerns an aquifer with a thickness of $50 \mathrm{~m}$, a hydraulic conductivity of $1 \mathrm{~m} /$ day, and a porosity of 0.3 . The dimensionless density difference was only 0.002 . The injection rate was $500 \mathrm{~m}^{3} /$ day for 100 days, followed by a storage period of 165 days and a recovery period of 100 days with the same rate as used for injection. Once the threshold concentration is reached, the system remains idle until the end of the recovery

Table 1 Ranges of the physical parameters of the problem

\begin{tabular}{ll}
\hline Parameter & Range \\
\hline Discharge $Q$ & $100-2,000 \mathrm{~m}^{3} /$ day \\
Hydraulic conductivity $k$ & $1-20 \mathrm{~m} / \mathrm{day}$ \\
Aquifer thickness $H$ & $5-50 \mathrm{~m}$ \\
Dimensionless density difference $v$ & $0.002-0.04$ \\
Porosity $n$ & $0.1-0.3$ \\
\hline
\end{tabular}

period. The specified longitudinal and transverse dispersivities are 0.3 and $0.03 \mathrm{~m}$, respectively. Calibration of variable density models of coastal aquifers to field data often lead to even smaller values for the dispersivity (e.g., Lebbe 1999). As the simulations by WEA are performed on a fixed two-dimensional grid, numerical dispersion is likely to occur as well, but was not reported.

The reported values yield a value of $D$ equal to 100 . For this value of $D$, the approach presented here yields a recovery efficiency for the first cycle of $52 \%$, which is approximately equal to the value that may be read from Fig. 9 of WEA. For cycle 5, the approach presented here yields a recovery efficiency of $81 \%$, which is significanlty higher than the $65 \%$ obtained by WEA. Cross-sectional plots of the results of WEA are presented in their Fig. 5 and show a significant effect of dispersion, as the transition zone is approximately $10 \mathrm{~m}$ thick. Whether this is caused by the specified dispersivity or numerical dispersion is unclear, but it seems likely that mixing is the major cause of the discrepancy between the two solutions.

One of the most interesting findings in WEA is that a numerical model with an equivalent homogeneous hydraulic conductivity value resulted in a recovery efficiency that is very similar to the recovery efficiency of the original layered system, even when the hydraulic conductivity contrast between layers was one or two orders of magnitude. This broadens the usefulness of the solution presented in this paper.

\section{Conclusions}

A new solution for radial Dupuit interface flow was presented to simulate the injection, storage and recovery of freshwater in saltwater aquifers. An equation was derived for the radial velocity of points on the interface 


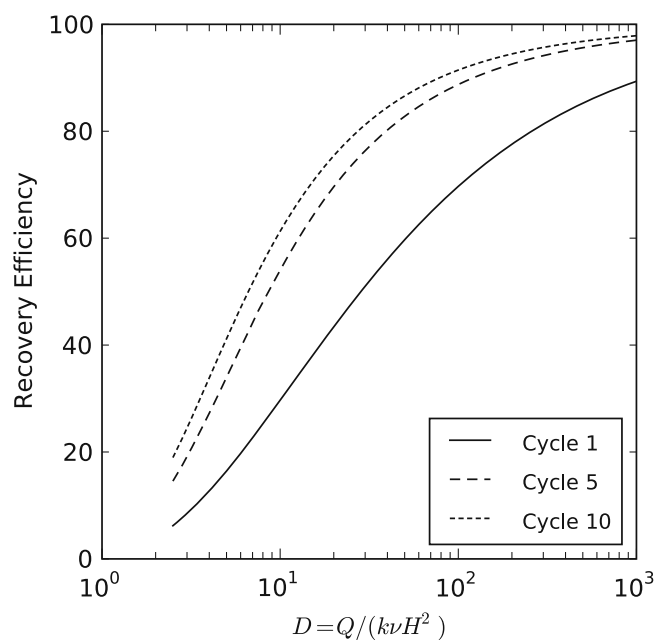

Fig. 5 Recovery efficiency as a function of $D=Q /\left(k v H^{2}\right)$ for the case without storage

as a function of the vertical coordinate and of time. The radial velocity is governed by only one dimensionless parameter $D=Q /\left(k v H^{2}\right)$ and by the relative lengths of the injection, storage and recovery periods; the recovery period generally consists of a period of pumping until the toe of the interface reaches the bottom of the well screen followed by an idle period. Furthermore, it was shown that the vertical position of the interface can be written as a function of the similarity variable $u=\rho^{2} / \tau$.

The practical meaning of these findings is that an ASR scenario of injection for 10 days, storage for 5 days and recovery for 10 days results in the exact same recovery efficiency as a scenario of 100 days of injection, 50 days of storage and 100 days of recovery, provided that the same value of $D$ is used. The actual position of the interface after 100 days of injection is, of course, different from the position after 10 days of injection; however, they are identical when the elevation of the interface is plotted as a function of $r^{2} / t$.

Formulation of the problem in terms of the radial velocity as a function of the vertical coordinate and time allows for the use of a fixed vertical grid to obtain a numerical solution. The numerical solution is relatively straightforward when the highly non-linear differential equation is written in the form of the diffusion equation, and the pseudo-source and diffusion terms are kept constant during a time step. A predictor-corrector approach is used to obtain accurate values for these pseudo terms during a time step, which results in accurate volume balances and short computation times.

Graphs were constructed for the recovery efficiency as a function of the parameter $D$ for several ASR scenarios. These graphs and the numerical solution are intended to be used as a screening tool to assess whether a specific ASR scenario results in acceptable recovery efficiencies. When acceptable values are obtained, additional modeling is warranted to assess the effects of other important factors such as mixing and chemistry.

Acknowledgements This research was funded in part by the Joint Water Research Program of the Dutch Water Supply Companies.

\section{Appendix}

A brief derivation is given of the relationships Eq. (20). Consider a monotonically increasing function $\zeta(\rho, \tau)$ (Fig. 7). As $\zeta$ is monotonically increasing, the same curve may be represented by the monotonically increasing function $\rho(\zeta, \tau)$. From Fig. 7 it may be seen that

$\frac{\partial \zeta}{\partial \rho}=-\frac{\partial \zeta}{\partial \tau}\left(\frac{\partial \rho}{\partial \tau}\right)^{-1}$

In the main body of the paper, the vertical position of the interface is called $\eta(\rho, \tau)$ rather than $\zeta(\rho, \tau)$, and the radial position $\sigma(\rho, \tau)$ rather than $\rho(\zeta, \tau)$. Hence, the derivatives of $\zeta$ in Eq. (32) with respect to $\rho$ and $\tau$ need to be replaced by derivatives of $\eta$ with respect to $\rho$ and $\tau$. Similarly, derivatives of $\rho$ with respect to $\zeta$ and $\tau$ need to be replaced by derivatives of $\sigma$ with respect to $\zeta$ and $\tau$. After these replacements, Eq. (32) may be converted directly into the first relationship of Eq. (20).
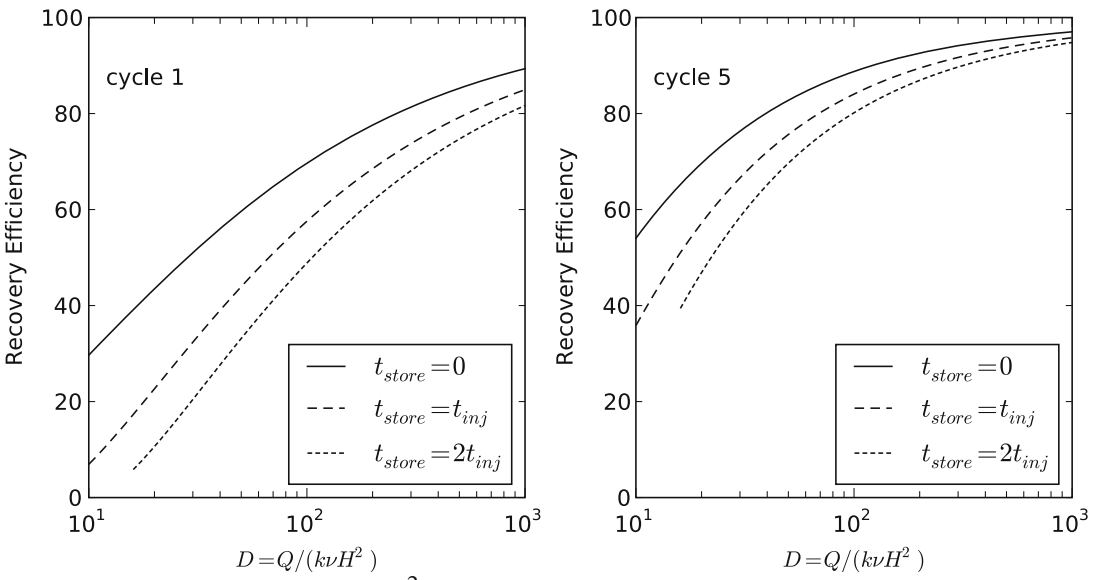

Fig. 6 Recovery efficiency as a function of $D=Q /\left(k v H^{2}\right)$ for three periods of storage 


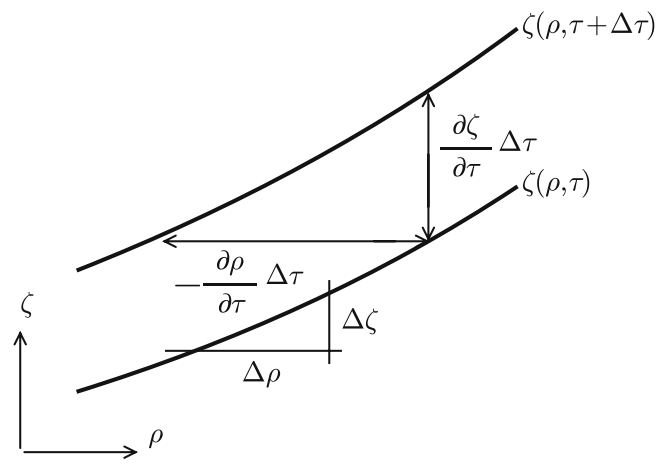

Fig. 7 Relating the derivatives of $\zeta(\rho, \tau)$ to the derivatives of $\rho(\zeta, \tau)$. Two heavy lines depict interface positions at $\tau$ and $\tau+\Delta \tau$.

From Fig. 7, it may be seen that

$$
\frac{\partial \zeta}{\partial \rho}=\left(\frac{\partial \rho}{\partial \zeta}\right)^{-1}
$$

This leads directly to the second relationship in Eq. (20) when the derivatives of $\zeta$ and $\rho$ are replaced by derivatives of $\eta$ and $\sigma$, respectively, as for Eq. (32). Differentiation of Eq. (33) gives

$\frac{\partial^{2} \zeta}{\partial \rho^{2}}=-\left(\frac{\partial \rho}{\partial \zeta}\right)^{-2} \frac{\partial}{\partial \zeta}\left(\frac{\partial \rho}{\partial \zeta}\right) \frac{\partial \zeta}{\partial \rho}$

so that, using Eq. (33),

$\frac{\partial^{2} \zeta}{\partial \rho^{2}}=-\left(\frac{\partial \rho}{\partial \zeta}\right)^{-3} \frac{\partial^{2} \rho}{\partial \zeta^{2}}$

The third relationship in Eq. (20) is obtained after the derivatives are replaced, as for Eqs. (32) and (33).

\section{References}

Arthur, Jonathan D, Dabous, AA, Cowart JB (2002) Mobilization of arsenic and other trace elements during aquifer storage and recovery, southwest Florida. US Geol Surv Open-File Rep 02-89

Bakker M (1998) Transient Dupuit interface flow with partially penetrating features. Water Resour Res. 34(11):2911-2918
Bakker M (2003) A Dupuit formulation for modeling seawater intrusion in regional aquifer systems. Water Resour Res. 39 (5):1-10. doi:10.1029/2002WR001710

Bakker M, Oude Essink GHP, Langevin CD (2004) The rotating movement of three immiscible fluids: a benchmark problem. J Hydrol. 287:271-9

Bear J, Cheng AH-D, Sorek S, Ouazar D, Herrera I (eds) (1999) Seawater intrusion in coastal aquifers: concepts, methods and practices. Kluwer, Norwell, MA

Cederstrom DJ (1947) Artificial recharge of a brackish water well, vol 14, No. 14. Virginia Chamber Commerce, Richmond, pp 31-73

Chan Hong JR, Van Duijn CJ, Hilhorst D, Van Kester J (1989) The interface between fresh and salt groundwater: a numerical study. IMA J Appl Math. 42:209-40

Cheng AH-D, Ouazar D (eds) (2004) Coastal aquifer management: monitoring, modeling and case studies. Lewis, New York

Holzbecher E (1998) Modeling density-driven flow in porous media: principles, numerics, software. Springer, New York

Lebbe L (1999) Parameter identification in fresh-saltwater flow based on borehole resistivities and freshwater head data. Adv Water Resour. 22(8):791-806

Lowry CS, Anderson MP (2006) An assessment of aquifer storage recovery using ground water flow models. Ground Water. 44 (5):661-7

Nordbotten JM, Celia MA (2006) Similarity solutions for fluid injection into confined aquifers. J Fluid Mech. 561:307-27

Post V, Kooi H, Simmons C (2007) Using hydraulic head measurements in variable-density ground water flow analyses. Ground Water. 45(6):349-53

Reese RS (2002) Inventory and review of aquifer storage and recovery in southern Florida. US Geol Surv Water Resour Invest Rep 02-4036

Strack ODL (1989) Groundwater mechanics. Prentice Hall, Englewood Cliffs, NJ

Strack ODL (1995) A Dupuit-Forchheimer model for three-dimensional flow with variable density. Water Resour Res. 31 (12):3007-17

SWIM (2009) Salt Water Intrusion Meeting website. www.swimsite.org. Cited 9 June 2009

Verruijt A (1980) The rotation of a vertical interface in a porous medium. Water Resour Res. 16(1):239-40

Ward JD, Simmons CT, Dillon PJ (2007) A theoretical analysis of mixed convection in aquifer storage and recovery: How important are density effects? J Hydrol. 343:169-86

Ward JD, Simmons CT, Dillon PJ (2008) Variable-density modelling of multiple-cycle aquifer storage and recovery (ASR): importance of anisotropy and layered heterogeneity in brackish aquifers. J Hydrol. 356:93-105

Weeks EP (2002) A Historical Overview of Hydrologic Studies of Artificial Recharge in the U.S. Geological Survey. In: Aiken GR, Kuniansky EL (eds) Artificial Recharge Workshop Proceedings Sacramento, CA, 2-4 April 2002. US Geol Surv Open-File Rep 02-89 